\title{
High-Speed GaN-Based Green Light-Emitting Diodes With Partially n-Doped Active Layers and Current-Confined Apertures
}

\author{
J.-W. Shi, J.-K. Sheu, C.-H. Chen, G.-R. Lin, and W.-C. Lai
}

\begin{abstract}
We demonstrate a high-speed GaN-based green light-emitting diode for plastic optical fiber (POF) communication applications. By using a combination of n-type doping and undoped $\mathrm{In}_{x} \mathrm{Ga}_{1-x} \mathrm{~N} / \mathrm{GaN}$ based multiple quantum wells (MQWs), and a 76- $\mu \mathrm{m}$-diameter current-confined aperture structure, we can obtain an extremely high electrical-to-optical (E-O) $3 \mathrm{~dB}$ bandwidth ( $\sim 330 \mathrm{MHz})$, which is limited by the spontaneous recombination lifetime of the MQWs. A reasonable coupled power $(\sim 264 \mu \mathrm{W})$ can be simultaneously achieved for a $2 \mathrm{~mm}$ in diameter POF with a 0.5 numerical aperture (NA).
\end{abstract}

Index Terms-GaN, LED, optical fiber.

\section{INTRODUCTION}

$\mathbf{H}$ IGH-SPEED red LEDs [1] often serve as key transmitter components for communication networks using plastic optical fibers (POFs). According to the media-oriented systems transport (MOST) standard they must run at a data rate of $22.5 \mathrm{Mb} / \mathrm{s}$ [2]. However, the optical bandwidth of the red operating window $(\sim 650 \mathrm{~nm})$ of the current commercially used polymethylmethacrylate (PMMA) POF is narrower, and the propagation loss higher $(0.125 \mathrm{~dB} / \mathrm{m}$ vs. $0.09 \mathrm{~dB} / \mathrm{m})$ than that of the high-performance POF system [3]. This operates under a PMMA loss minimum window, which has a wavelength of around $500 \mathrm{~nm}$ [3]. The III-nitride-based green LEDs would thus be a more promising choice for such applications [4]. Although the material dispersion of the PMMA fiber at around $520 \mathrm{~nm}$ wavelength is higher (1.5 times higher) than at $650 \mathrm{~nm}$ wavelength, the dispersion-free window in the total dispersion spectrum, which includes the waveguide dispersion and the material dispersion, may be shifted to $520 \mathrm{~nm}$ by optimizing the index profile of the PMMA fiber [5]. In this letter, we demonstrated an $\operatorname{In}_{x} \mathrm{Ga}_{1-x} \mathrm{~N} / \mathrm{GaN}$-based high-speed green LED with a current-confined aperture and partial n-type doping $\mathrm{In}_{x} \mathrm{Ga}_{1-x} \mathrm{~N} / \mathrm{GaN}$-based multiple quantum wells (MQWs).

Manuscript received August 17, 2007; revised November 15, 2007. The review of this letter was arranged by Editor P. Yu.

J.-W. Shi and C.-H. Chen are with the Department of Electrical Engineering, National Central University, Taoyuan 320, Taiwan, R.O.C. (e-mail: jwshi@ ee.ncu.edu.tw; puq77@yahoo.com.tw)

J.-K. Sheu and W.-C. Lai are with the Institute of Electro-Optical Science and Engineering, Advanced Optoelectronic Technology Center, National Cheng Kung University Tainan 70101, Taiwan, R.O.C. (e-mail: jksheu@mail. ncku.edu.tw; weilai@mail.ncku.edu.tw).

G.-R. Lin is with the Graduate Institute of Electro-Optical Engineering, Department of Electrical Engineering, National Taiwan University, Taipei 10617, Taiwan, R.O.C. (e-mail: grlin@ntu.edu.tw)

Color versions of one or more of the figures in this letter are available online at http://ieeexplore.ieee.org.

Digital Object Identifier 10.1109/LED.2007.914070
With this device, one can achieve an extremely high electricalto-optical (E-O) 3-dB bandwidth ( $2330 \mathrm{MHz}$ ), limited only by the spontaneous lifetime of our MQWs, with a reasonable POF coupling power $(\sim 264 \mu \mathrm{W})$ suitable for POF communications.

\section{Device Structure AND FABricAtion}

The LED wafers were grown by metal-organic chemical vapor deposition (MOCVD) on a sapphire substrate. The active region of the LED was a ten-period $\operatorname{In}_{x} \mathrm{Ga}_{1-x} \mathrm{~N} / \mathrm{GaN}$ based MQW structure, which consists of a $135-\AA$-thick GaN barrier layer and a 25 - $\AA$-thick $\operatorname{In}_{x} \mathrm{Ga}_{1-x} \mathrm{~N}$ well layer in each period. The n-type doping density was around $5 \times 10^{17} \mathrm{~cm}^{-3}$ in seven of the GaN barrier layers near the n-type cladding layer. Conversely, the barrier layers near the p-type cladding layer were left undoped. Although n-type doping in the $\operatorname{In}_{x} \mathrm{Ga}_{1-x} \mathrm{~N} / \mathrm{GaN}$ based MQW layers can improve the material quality and the radiative recombination rate [4] in MQWs, this approach may result in most of the depletion region being located in the p-type $\mathrm{GaN}$ layers as the doping density in the MQW layers is comparable to the typical ionized doping density of the p-type $\mathrm{GaN}$ layer [6]. By inserting three undoped MQW periods near the p-side of the active region, the external electric field can be concentrated more in the active layers than is the case when fully n-type doped MQWs are used in the active layers. Compared with GaN LEDs with fully n-doped MQWs [4] under the same bias voltage, higher output power performance of demonstrated partially n-doped LEDs can, thus, be expected. For high-speed applications, a current-confined aperture was realized by etching a small mesa on the topmost p-type GaN layer to restrict the current-flow path and reduce the capacitance [1], [7]. The use of a current-confined aperture can eliminate etching-induced damage on the MQW sidewall and its influence on the internal quantum efficiency of LEDs, such as what occurs with direct etching through the active MQW layers to reduce capacitance. A conceptual cross-sectional view of demonstrated device and the top view of the current-confined aperture is given in Fig. 1 and its inset. In order to study the influence of the diameter of the current-confined aperture on the speed and output power performance of the LEDs, devices with different diameters of current-confined aperture and active areas were fabricated. The epilayers and geometric structure of devices A-D were similar, with only the ratio of the diameter of the current-confined aperture to that of total circular active mesa of the bottom side being varied. The ratios of devices A and B are 1:1 and 1:2, respectively. For devices $\mathrm{C}$ and $\mathrm{D}$, the ratio is the same at 1:3. The diameter of the circular active area below the p-type $\mathrm{GaN}$ 


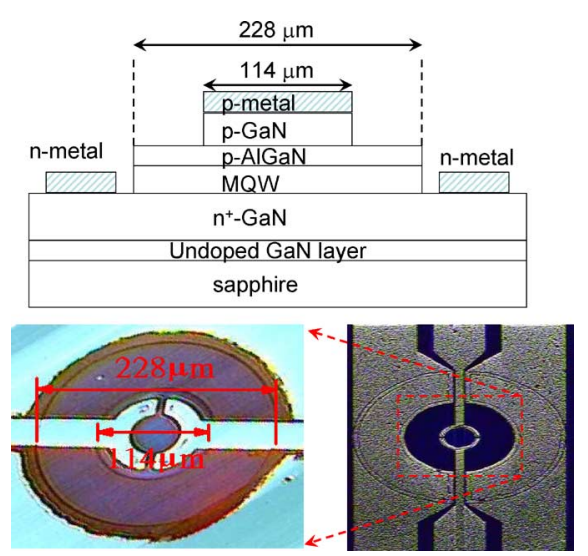

Fig. 1. Conceptual cross-sectional view of the demonstrated LED. The inset shows the top view of device after zooming in on the active current-confined aperture. For clearness, the figure was not drawn according to scale.

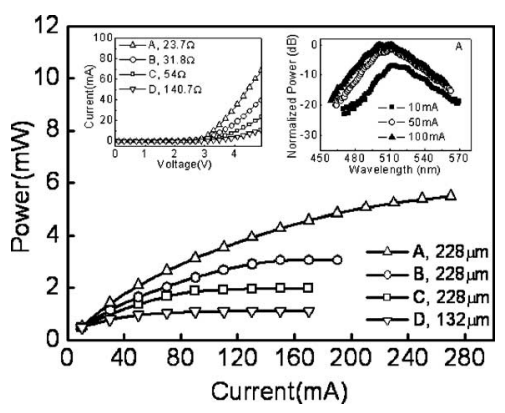

Fig. 2. Output optical power $(P)$ versus the bias current $(I)$ of the devices (A-D) measured with different diameters of current-confined apertures. The two insets show the measured $I-V$ curves of devices A-D and electroluminescence (EL) spectra of device A measured under different bias currents.

layer is $228 \mu \mathrm{m}$, the same as for devices A-C. Device D has a diameter of $132 \mu \mathrm{m}$. As shown in Fig. 1, the LED unit was integrated with two coplanar waveguide (CPW) pads [4], which were parallel to the uniform current distribution during the $\mathrm{dc}$ measurement. Alternatively,for the ac measurement, one of the CPW pads was left as RFs open. Details of the fabrication processes of the demonstrated devices can be referred to in our previous paper [4].

\section{MEASUREMENT RESULTS AND DISCUSSION}

During dc measurement, the output power of the LED was collected by use of an integrating sphere or a POF ( $2 \mathrm{~mm}$ in diameter and with a $0.485 \mathrm{NA}$ ), which served as an optical probe. All four devices exhibited similar bias-dependent electroluminescence (EL) spectra behavior with a similar center wavelength ( $\sim 510 \mathrm{~nm})$. The inset of Fig. 2 shows the EL spectra of device A measured under different bias currents. A slight blue shift of the center wavelengths $(\sim 510$ to $\sim 500 \mathrm{~nm})$ was observed when the bias current increased from 10 to $100 \mathrm{~mA}$, which can be attributed to the screening of the piezoelectric field and the renormalization of the quantized states in the well region under a forward bias [4]. Another inset of Fig. 2 shows the currentvoltage $(I-V)$ curves of devices A-D. The turn-ON voltages of these four devices were all around $\sim 3 \mathrm{~V}$. The values of the differ- (a)
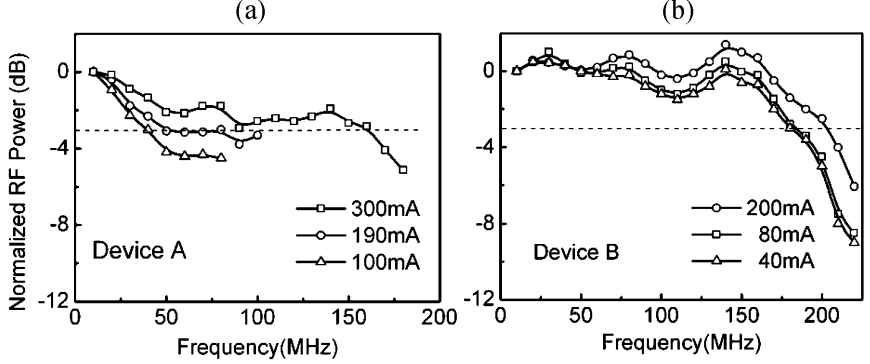

Fig. 3. E-O frequency responses of (a) device A and (b) device B measured under different bias currents.

ential resistances of each device are also specified in this inset. We can clearly see, via the difference in the cross-sectional areas of the current-flowed paths, that device A has a much lower differential resistance than device D. Fig. 2 shows the optical power $(P)$ collected by use of the integrating sphere versus the injected current $(I)$ for the four devices. We can clearly see that device $A$ has a much higher saturation output power than that of devices B-D. This is due to their much smaller light-emitting area, much larger differential resistance, as shown in the inset to Fig. 2, and more serious device heating effect than device A. By measuring the difference in collected optical power, obtained using an integrating sphere and a POF, we can obtain the coupling efficiency of the devices: this was the same for all four devices, around $13.4 \%$, which is close to the reported coupling efficiency $(\sim 11 \%)$ for high-speed $\mathrm{GaN}$ based green LEDs to POFs [3]. The similar coupling efficiency of all of our devices can be ascribed to the fact that their output far field is similar and can be approximated by an ideal Lambertian farfield distribution regardless of the diameter of the light-emitting aperture [8]. Even device C, with its small light-emitting area, had a coupled optical power $(185 \mu \mathrm{W}$ under $50 \mathrm{~mA})$ to the POF large enough for POF communication [3]. Using the same measurement setup and the same bias current $(\sim 100 \mathrm{~mA})$ as in our previous paper, we found the POF-coupled optical power of the current LED (device B) to be much higher $(343 \mu \mathrm{W}$ vs. $170 \mu \mathrm{W})$ than that of our previous fully n-type doped MQW green LED, even though it had a larger active area $\left(\sim 14000 \mu \mathrm{m}^{2}\right.$ vs. $\sim 10000 \mu \mathrm{m}^{2}$ ) [4]. As can be seen in Fig. 2, the $P-I$ measurement results are superior to those found in our previous paper [4], which implies the advantages of using partially n-doped MQW structures. During the ac measurement, we injected the RF signal into the LEDs. The modulated optical power was collected by the POF and then fed into a low-noise Si-based photoreceiver (New Focus: 1801) that was connected with an RF spectrum analyzer. We only deembedded the frequency response of the photoreceiver because the microwave loss for the $\mathrm{K}$-band passive RF components used in the frequency range of interest (hundreds of megahertz) for LED measurement is negligible. Fig. 3(a) and (b) and Fig. 4(a) and (b) show the measured E-O frequency responses under different bias currents obtained for devices A-D. The maximum bias current is limited by device failure. We can clearly see that the measured $3 \mathrm{~dB}$ bandwidth of each device increases significantly with a bias current density. This phenomenon has been reported for other LEDs and 
(a)

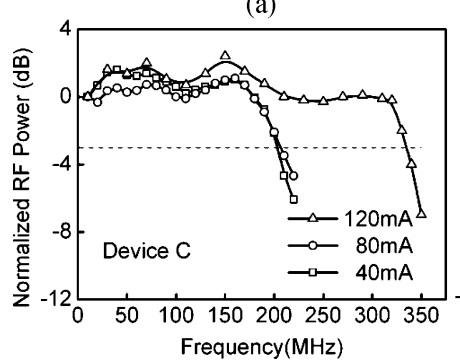

(b)

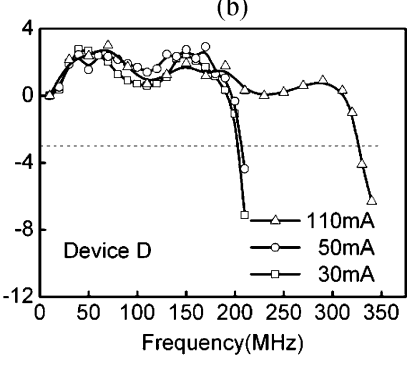

Fig. 4. E-O frequency responses of (a) device $\mathrm{C}$ and (b) device D measured under different bias currents.

can be attributed to the bimolecular recombination probability being proportional to the injected carrier density into the active volume [8], [9]. Devices $C$ and D have a bias current of around $100 \mathrm{~mA}$ and a very high current density $\left(>2.2 \mathrm{kA} / \mathrm{cm}^{2}\right)$, and both could exhibit a $3 \mathrm{~dB}$ bandwidth as high as $330 \mathrm{MHz}$. The coupled optical power of device $\mathrm{C}$ under high-speed operation was around $264 \mu \mathrm{W}$. To the best of the authors' knowledge, this is the highest $3-\mathrm{dB}$ modulation bandwidth $(330 \mathrm{MHz})$ ever reported for III-nitride-based LEDs [3], [4]. Such a value is comparable with the highest reported $3 \mathrm{~dB}$ bandwidth $(300-500 \mathrm{MHz})$ for AlGaInP-based red resonant cavity LEDs (RCLEDs) [1]. There are two major factors limiting the speed of LEDs: one is the $R C$ time constant and the other is the spontaneous recombination time. In order to clarify which one caused the significant bandwidth enhancement observed for devices $C$ and $D$, we performed capacitance-voltage $(C-V)$ and current-voltage $(I-V)$ measurements to obtain the $R C$-limited bandwidths of the devices. The extracted $R C$-limited bandwidth, which includes the $50-\Omega$ source resistance, of devices A, C, and D were around $150 \mathrm{MHz}, 1 \mathrm{GHz}$, and $3 \mathrm{GHz}$, respectively. Such a result indicates that the current-confined aperture can effectively reduce the diffusion capacitance and improve the $R C$-limited bandwidths. According to the extracted $R C$-limited and measured $3 \mathrm{~dB}$ bandwidths of the devices, we can conclude that under a high bias current density, the dominant bandwidth limiting factor of device $\mathrm{A}$ is the $R C$ delay time, and for devices $\mathrm{C}$ and $\mathrm{D}$, it is the spontaneous recombination time and the maximum allowable bias current density. A higher speed performance (near $1 \mathrm{GHz}$ ) [1], [9], [10] may be expected by decreasing the thickness of the active layer (MQW) (by increasing the injected carrier density) [1], [9], [10], using the fully doped MQWs structure at the expense of lower output power [1], [4], [10], or further minimizing the device resistance and heating effect to increase the maximum allowable bias current density. Based on the measured $3 \mathrm{~dB}$ bandwidth $(330 \mathrm{MHz})$, the extracted recombination time will be around $0.5 \mathrm{~ns}$ [9], which is a reasonable number and comparable to the measurement results $(\sim 1 \mathrm{~ns})$ for fully n-type doped InGaN/GaN-based MQWs obtained using the time-resolved photoluminescence technique [11].

\section{CONCLUSION}

We demonstrate a GaN-based high-speed green LED. By utilizing a current-confined aperture with partially n-doped MQW active layers, we can simultaneously achieve a 330-MHz 3-dB E-O bandwidth with a reasonable $(\sim 264 \mu \mathrm{W})$ POF optical coupling power. Such an excellent high-speed performance can be attributed not only to an increase of the bias current density but also to a reduction of device capacitance through the use of the current-confined structure.

\section{REFERENCES}

[1] R. Wirth, B. Mayer, S. Kugler, and K. Streubel, "Fast LEDs for polymer optical fiber communication at $650 \mathrm{~nm}$," in Proc. SPIE, vol. 6013 , Bellingham, WA, 2005.

[2] MOST Cooperation, Specification Rev. 2.4, May 2005.

[3] M. Akhter, P. Maaskant, B. Roycroft, B. Corbett, P. de Mierry, B. Beaumont, and K. Panzer, "200 Mbit/s data transmission through $100 \mathrm{~m}$ of plastic fiber with nitride LEDs," Electron. Lett., vol. 38, pp. 1457-1458, Nov. 2002.

[4] J.-W. Shi, H.-Y. Huang, J.-K. Sheu, C.-H. Chen, Y.-S. Wu, and W.-C. Lai, "The improvement in modulation speed of GaN-based light-emitting diode (LED) by use of n-type barrier doping for plastic optical fiber (POF) communication," IEEE Photon. Technol. Lett., vol. 18, no. 15, pp. 16361638, Aug. 2006

[5] G. Yabre, "Theoretical investigation on the dispersion of graded-index polymer optical fibers," J. Lightw. Technol., vol. 18, no. 6, pp. 869-877, Jun. 2000.

[6] S. Nakamura, M. Senoh, and T. Mukai, "Highly P-typed Mg-doped GaN films grown with GaN buffer layers," Jpn. J. Appl. Phys., vol. 30, no. 2, pp. L1708-L1711, Oct. 1991.

[7] M. Kneissl, T. L. Paoli, P. Kiesel, D. W. Treat, M. Teepe, N. Miyashita, and N. M. Johnson, "Two-section InGaN multiple-quantum-well laser diode with integrated electro-absorption modulator," Appl. Phys. Lett., vol. 80, pp. 3283-3285, May 2002.

[8] P. Bhattacharya, Semiconductor Optoelectronic Devices. Taipei, Taiwan: Pearson Education, 2003, pp. 216-218.

[9] K. Ikeda, S. Horiuchi, T. Tanaka, and W. Susaki, "Design parameters of frequency response of GaAs- $(\mathrm{Ga}, \mathrm{Al}) \mathrm{As}$ double heterostructure LED's for optical communications," IEEE Trans. Electron Devices, vol. ED-24, no. 7, pp. 1001-1005, Jul. 1977.

[10] R. Windisch, A. Knobloch, M. Kuijk, C. Rooman, B. Dutta, P. Kiesel, G. Borghs, G. H. Döhler, and P. Heremans, "Large-signal-modulation of high-efficiency light-emitting diodes for optical communication," IEEE J. Quantum Electron., vol. 36, no. 12, pp. 1445-1453, Dec. 2000.

[11] M. S. Minsky, S. Chichibu, S. B. Fleischer, A. C. Abare, J. E. Bowers, E. L. Hu, S. Keller, U. K. Mishra, and S. P. Denbaars, "Optical properties of InGaN/GaN quantum wells with Si doped barriers," Jpn. J. Appl. Phys., vol. 37, no. 2, pp. L1362-L1364, Nov. 1998. 